\title{
Faire le point sur l'urbanisme tactique : entre innovations et dérives dans la fabrique de la ville
}

\author{
Sylvain Lefebvrea ${ }^{a}$ Jérémy Diaz ${ }^{b}$, Jean-Marc Adjizianc
}

RÉSUMÉ. L’article s'intéresse au phénomène très répandu ces dernières années de «l'urbanisme tactique », une tendance qui recoupe plusieurs réalités et nouveaux types d'aménagement et de planification territoriale. Ces nouvelles modalités de fabrique de la ville peuvent représenter un amalgame de pratiques très diversifiées de réappropriation de l'espace urbain, privé ou public, se traduisant par des transformations physico-spatiales ou événementielles, généralement spontanées, citoyennes et politiquement engagées (urbanisme temporaire, éphémère, populaire, insurgé, transitoire, etc.). Elles prennent d'assaut les interstices de l'espace urbain, sont parfois illégales (l'idée de guérilla urbaine, de space hacking, etc.), puis sont récupérées par les instances (administrations locales, promoteurs, investisseurs) comme prévalorisation au marché foncier et aux espaces environnants. Montréal n'a pas échappé à ce phénomène, et la multiplication des projets et des initiatives est exponentielle depuis quelques années.

\begin{abstract}
This article presents issues about a popular urban phenomenon called «tactical urbanism 》, a generic term used to describe different kinds of urban projects and new ways of making urban spaces. These new practices are often translated to ways of reclaiming private and public spaces, are sometimes event-oriented, and are tainted with a strong community-based ideology. Temporary and transitory uses, urban guerillas, space hacking, yarn or seed bombings, park(ing) days, etc., are a few examples of tactical urbanism projects which are gradually interfering with more traditional or normative urban planning processes. It is sometimes used by private developers or public officials to pre-vitalize some new urban projects, and many new challenges are now rising with the multiplication of these initiatives spreading all over in the neighbourhoods and cities. Montreal is an interesting case study in this regard.
\end{abstract}

\section{Introduction}

Les modes de production de l'espace urbain évoluent rapidement depuis trois décennies. Sont apparus de nouvelles pratiques et de nouveaux acteurs qui revendiquent le droit de produire et de transformer nos milieux de vie. Dans ce contexte, le mot clé qui est venu influencer le discours de l'urbanisme régulateur et politique est désormais celui de «réappropriation». Réappropriation des espaces publics, des rues, des berges, des espaces naturels, des friches, des espaces vacants, etc. Par ailleurs, ce terme est aussi revendiqué par des organisations locales, communautaires, des groupes d'intérêt, des regroupements de citoyens et des individus isolés. Devant les menaces que font peser les grands projets urbains et les opérations de revitalisation ou de mise en valeur de différents types de quartiers (provoquant de l'embourgeoisement, de nouvelles mixités commerciales, etc.), les mobilisations citoyennes et les innovations urbanistiques ont pris des formes inusitées qui séduisent plusieurs instances de planification territoriale et l'opinion publique en général. Au centre de ce phénomène, l'urbanisme tactique est rapidement devenu un incontournable.

On définit l'urbanisme tactique en plusieurs déclinaisons issues du mouvement Do it yourself (DIY), plus largement utilisé dans la littérature anglophone sous l'appellation « urbanisme DIY ». Il se caractérise principalement par des actions de transforma-

\footnotetext{
a Professeur, département de géographie, Université du Québec à Montréal

b Doctorant, département d'études urbaines et touristiques, Université du Québec à Montréal

c Doctorant, département de l'activité physique, Université d'Ottawa
} 
tion de l'espace urbain qui sont généralement individuelles (ou instaurées par de petits groupes de personnes), spontanées, innovatrices et à faible coût. On y retrouve une large gamme de projets pouvant s'apparenter à une forme d'acupuncture urbaine (jardins spontanés et verdissement de microespaces, installations temporaires de mobilier urbain et/ou d'œuvres artistiques, embellissement de la voirie et des espaces dégradés), mais aussi à une sensibilisation accrue sur des enjeux de « droit à la ville» (occupation illégale ou détournée de l'espace privé et/ou public, revendications diverses par l'affichage ou l'aménagement d'artéfacts sans permis ou autorisation formelle). Ces formes d'urbanisme DIY sont parfois prises en charge par des organismes à but non lucratif (OBNL) qui multiplient leurs actions en concertation avec divers acteurs privés et publics en reproduisant des aménagements innovants et adaptés aux besoins des territoires et des usagers.

Se multiplient ainsi des lieux en site propre où des conteneurs sont recyclés pour être reconvertis en bars ou cafés à ciel ouvert, en commerces ou espaces d'exposition, le tout cohabitant avec du mobilier urbain temporaire (tables, bancs, fauteuils, etc.) fabriqué avec des matériaux recyclés ou récupérés à moindres frais (palettes de bois, contenants, paniers de métal), des espaces de détente et de divertissement (petites scènes de spectacle et pistes de danse), des œuvres d'art et des microespaces d'expression et de jeu pour enfants, des camionsrestaurants, un éclairage festif et les incontournables parcelles et bacs d'agriculture urbaine, de verdissement et autres minipotagers.

Existent aussi des micro-interventions plus discrètes sur les trottoirs et les rues, dont l'embellissement des carrés d'arbre, des interstices sur la chaussée (guerilla gardening ou seed bombing), des pianos publics libres d'accès, des croque-livres (ou boittes à livres gratuits), de même que des gestes artistiques spontanés (murales; graffitis; petits artéfacts sur les arbres, les façades, dans les ruelles; tricots-graffitis ou yarn bombing), etc. Le tout, conjugué à toutes les sauces, offre des ambiances de détente, de déambulation, de "pause urbaine » et d'îlots de résistance dans ces lieux abîmés, mal-aimés ou en chantier.

Les rythmes créés dans les sites les plus établis et reconnus semblent accommoder un amalgame de profils types d'usagers, qui vont de la population du quartier touché aux curieux et visiteurs de passage et, bien sûr et surtout, aux jeunes professionnels créatifs en mal d'espaces innovants et expérientiels, le tout dans une saveur ludique, festive et bohémienne. Il y a plusieurs autres espaces (parcs, rues commerçantes, etc.) en bon état et plus conventionnels qui font aussi l'objet d'interventions tactiques. Ainsi, les lieux de destination générés pour un type ciblé d'usagers se distinguent sensiblement des lieux de proximité, intergénérationnels, créés au cœur des quartiers.

\section{Les origines du phénomène}

Qu'est-ce que l'urbanisme tactique? Parmi les définitions proposées par une multitude d'auteurs, quatre éléments représentent les dénominateurs communs de la grande diversité des projets et des actions relevant généralement de ce type d'urbanisme et d'aménagement du territoire: l'échelle relativement restreinte du lieu touché, le faible coût de l'aménagement et/ou de l'opération, la temporalité relativement courte du projet et la dimension citoyenne dans l'initiative proposée (Lydon et Garcia, 2015; Ferraris, 2016; Talen, 2015)ํ.

Se déclinent par la suite plusieurs autres caractéristiques de cet urbanisme tactique selon la nature des interventions, des milieux et territoires visés, des acteurs initiateurs de ces projets ou encore les motivations ou objectifs poursuivis par ces derniers. Cette diversité introduit un peu de confusion puisqu'elle élargit les finalités et les possibles configurations des actions et des initiatives. Entre un projet modeste instauré par un seul citoyen tout près de son domicile et une stratégie plus complexe, dispersée sur un territoire et menée par un groupe de pression ou un OBNL, il y a un éventail impressionnant de possibilités.

L'urbanisme tactique se distingue du cadre normatif en urbanisme (les traditionnels projets d'aménagement, plans et schémas, règlements et autres relevant d'une approche descendante ou top-down) par une prise en charge individuelle ou collective d'initiatives spontanées, parfois concertées, pour pallier la lenteur et la rigidité des systèmes plus traditionnels de planification territoriale. Ainsi, cette approche ascendante (bottom-up) de l'urbanisme tactique est souvent perçue comme citoyenne, émancipatrice (empowerment), avantgardiste et expérimentale, permettant des ajustements aux prototypes originaux de même que des 
adaptations en fonction de la réelle utilisation de ces nouveaux lieux. La promotion de solutions temporaires ou légères est ainsi justifiée par la possibilité de faire des tests, d'expérimenter et d'adapter ces solutions pour satisfaire des projets durables et plus efficaces (Silva, 2016).

En marge des structures établies, ces interventions bousculent les manières de faire la ville, révélant les possibles de secteurs urbains en mal d'amour. [...] Ancrées dans l'instantané et l'éphémère, [elles] agissent comme un baume sur l'espace public, redonnant à des lieux dépréciés une nouvelle vocation pour un temps. Gentiment subversif et fondamentalement durable [...], l'urbanisme tactique se veut aussi une manière pour les citoyens de se réapproprier les lieux d'expression de leur quotidien. (Ferraris, 2016, s. p.)

On accorde parfois au mouvement situationniste (1957-1972) de Guy Debord quelques fondements au mouvement social qu'est devenu l'urbanisme tactique. Ce mouvement a créé les premières balises d'une psychogéographie dénonçant le conformisme et les dysfonctionnements de l'urbanisme fonctionnaliste. Les situationnistes contestent le conservatisme social et le virage moderniste de l'architecture de cette époque en privilégiant d'autres options aux modèles du travail et de l'économie capitaliste, en proposant aussi l'altérité et la singularité, plutôt que la reproduction de solutions prédéterminées dans la ville (Finn, 2014).

En suggérant une occupation de l'espace et du territoire par l'intermédiaire de détournements, de jeux, de loisirs, de surprises (des « situations »), de l'improvisation et d'interventions artistiques, les situationnistes participent ainsi à un renversement des valeurs dominantes et à une transformation radicale de leur milieu de vie (Paquot, 2015). Les situationnistes prônent diverses formes d'activisme social, des gestes artistiques subversifs et des pratiques qui sont au plus près de celles des projets d'urbanisme tactique du début des années 1990. On y trouve donc les mêmes valeurs de rejet et d'avantgardisme qui viennent teinter les multiples déclinaisons depuis, plus récentes : urbanisme temporaire, urbanisme interstitiel, ville éphémère ( urbanisme populaire (grassroots), urbanisme guérilla, urbanisme insurgé (insurgent), piratage urbain (backing), urbanisme du quotidien, urbanisme improvisé, urbanisme fait main (handmade), urbanisme spontané, urbanisme fait maison (DIY), ville qui se fait toute seule (self-made) (Nédélec, 2017).

On pourrait croire aussi que les exemples récents donnés pour illustrer ce qu'est l'urbanisme tactique sont plutôt originaux et nouveaux. Ce n'est pas le cas. L'installation de diverses formes de verdissement ou de micromarchés dans les lieux abandonnés, de bancs publics ou de jeux pour enfants dans des espaces vacants, la réutilisation de lieux sousutilisés et la mise en place informelle et illégale d'activités marchandes, de divertissement, etc. dans l'espace public constituent un phénomène décrit différemment dans les pays en développement dans le registre des activités informelles (Talen, 2015; Acuto, Dinardi et Marx, 2019).

L'occupation du foncier est motivée par une logique différente, mais les modalités de réappropriation restent les mêmes dans la plupart des cas : le caractère informel dans la fabrique de la ville y est autorisé ou toléré, malgré un cadre règlementaire et normatif préexistant. Le «droit à la ville» y côtoie ainsi la « ville de droit». En d'autres termes, les mécanismes formels de planification territoriale nécessaires pour coordonner et harmoniser le développement urbain ainsi que les moyens légaux et normatifs qui en découlent s'accommodent d'une prolifération d'occupations plus ou moins légitimes, mais nécessaires ou indispensables à la population concernée. Toutefois, la grande dispersion et le caractère parfois chaotique de ces occupations créent des tensions importantes avec la gestion et l'harmonisation du développement urbain. Nous le verrons plus loin, les tensions entre légalité et légitimité sont au centre des enjeux suscités par l'urbanisme tactique.

Ces pratiques informelles sont donc plutôt anciennes et standardisées dans les pays en développement, alors que, dans les pays industrialisés, elles apparaissent innovantes et révolutionnaires (Talen, 2015). Elles relancent aussi un débat épistémologique dans les champs des études culturelles, de la géographie, des études urbaines et des sciences politiques sur la capacité de cet urbanisme informel non pas de réformer, mais d'agir en catalyseur des changements dans les théories sur la ville (Acuto et collab., 2019). Cette informalité reste un dénominateur commun de toutes les interventions d'urbanisme tactique si et seulement si les acteurs à l'origine de ces dernières n'ont pas de lien avec le cadre 
normatif, formel ou décisionnel en aménagement du territoire (Ferraris, 2016). Or, comme nous le verrons plus tard, les projets de cet urbanisme tactique proviennent dorénavant de tous les horizons et secteurs, nonobstant la nature des acteurs concernés.

On retrouve aussi des traces d'urbanisme tactique en 1914 dans la ville de New York avec le programme Play Streets, qui est l'ancêtre des projets Open Streets de la ville de Seattle en 1965 et de Atlanta Streets Alive, des projets essentiellement de réappropriation citoyenne, ludique et festive de certaines rues (Silva, 2016). Les villes de Bogota en Colombie ainsi que de Sao Paulo et Curitiba au Brésil ont aussi eu des projets de réappropriation temporaire des rues dans les années 1970 pour les réserver aux cyclistes, piétons et autres utilisateurs non motorisés (première piste cyclable instaurée en 1974 à Bogota, puis reconduite périodiquement, ainsi que des événements organisés plus tard dans les villes colombiennes de Cali et Medellin).

Plus récemment, dans les villes des pays industrialisés, le phénomène de l'urbanisme tactique s'est réactualisé en "mouvement» ou en nouvelle tendance. On attribue à certains collectifs d'artistes ou groupes de citoyens de la ville de San Francisco les premières initiatives en 2004 et 2005, dont les park.(ing) days ${ }^{2}$, journées dédiées qui consistent à payer la tarification d'un espace de stationnement sur rue, mais d'y aménager, pour la durée tarifée, un espace vert, de détente, de jeu ou encore une œuvre d'art ou un événement afin de sensibiliser la population à la réappropriation de l'espace public et des espaces de stationnement sur rue pour diverses finalités autres que celles liées au transport motorisé. En continuité avec cette logique, plusieurs commerçants, voire les autorités locales ont pu aménager des espaces plus permanents, des parklets (terrasses, microespaces publics, etc.), à même certains espaces de stationnement sur rue, en saison touristique ou pour une période à moyen et long terme afin de rendre les rues plus animées et conviviales sur des espaces bien ciblés et stratégiques.

Cet urbanisme est aussi parfois qualifié open source, c'est-à-dire un urbanisme de mise en commun, de partage de « données » ouvertes, accessibles, gratuites, mais aussi de plusieurs autres éléments concrets (mobilier urbain, matériaux recyclés, artéfacts récupérés de diverses sources). Cette production de « communs urbains » est vite devenue aussi une caractéristique des interventions d'urbanisme tactique (Bradley, 2015). Les valeurs sous-jacentes à ces communs urbains reposent principalement sur celles d'un mouvement plus élargi fondé sur une distribution plus équitable du pouvoir, des savoir-faire et des moyens de production :

Open-source modes of production are not only in use by hackers and civil society groups, but also by public authorities. [...] Urban commons, and, more precisely, groups that operate using open-source tactics, should not necessarily be interpreted as expressions of "anti-statism" but rather as methods beneficial and useful to public authorities as well. (Bradley, 2015, p. 92)

De même, plusieurs pratiques et plateformes participatives en matière d'urbanisme tactique ne contribuent pas directement à la lutte aux inégalités. Elles reproduisent plutôt les rapports de force qui préexistaient aux différents projets. Les exemples pionniers des park(ing) days et des parklets de San Francisco sont éloquents à cet égard : les autorités locales ont rapidement instauré des moments précis pour la tenue de ces événements, qui relevaient à l'origine d'une "guérilla urbaine » dans sa forme la plus épurée. Pour les parklets, des permis d'occupation temporaire de certains espaces sur rue ont été délivrés par souci de bien encadrer et d'harmoniser les aménagements saisonniers et/ou éphémères. L'évolution du cycle de vie de certains projets d'urbanisme tactique semble ainsi se reproduire dans plusieurs villes de façon plutôt uniforme. Les actions spontanées, «hors-la-loi», improvisées, expérimentales et innovantes de ces initiatives alimentent peu à peu un véritable mouvement social qui se généralise, puis elles se retrouvent incorporées aux pratiques plus formelles et institutionnalisées de la planification territoriale.

\section{Les enjeux contemporains}

Malgré l'engouement généralisé pour les diverses manifestations de l'urbanisme tactique, une série de questions se présente sur les possibles dérapages, problèmes ou enjeux qui en découlent au fil de son évolution. Le principal enjeu remet en question la légitimité des actions prises dans ces espaces publics, parfois semi-publics ou même privés. Jusqu'où peut-on permettre à quiconque de décider de la configuration, de la localisation et du design final d'un projet d'urbanisme tactique? Il n'existe 
aucune méthode objective, consensuelle ni reconnue pour guider les instigateurs de projets ainsi que les modalités de mise en place, de gestion et de suivi de ces interventions. La multiplication excessive des interventions DIY sans cohérence d'ensemble, sans vision globale et surtout sans critères ou balises d'intervention est peu à peu critiquée par certains auteurs, qui tentent de nuancer ce « droit à la ville » non organisé ou non orchestré (Talen, 2015; Silva, 2016; Spataro, 2015; Deslandes, 2013).

De même, ce souci pour des projets de petite taille, nécessitant peu de ressources et modestes dans leur configuration finale et dans les modalités de leur mise en place séduit passablement les tenants d'une "ville à l'échelle humaine », de cette « acupuncture urbaine » perçue comme une médecine douce et naturelle qui soulage et apaise l'espace urbain. Ce réflexe de résistance locale devant un urbanisme fonctionnaliste et moderniste semble faire toutefois l'économie d'une réflexion plus systématique sur les échelles plus globales, qu'elles soient métropolitaines ou nationales (Banville, 2016).

Par ailleurs, certains auteurs observent avec justesse que l'urbanisme tactique véhicule une image « branchée» et une esthétique de créativité qui sont largement récupérées par l'agenda néolibéral des administrations locales et des promoteurs dans les opérations de revalorisation et de revitalisation de quartiers en transition (Mould, 2014; Courage, 2013). Ce faisant, les processus d'embourgeoisement et les stratégies de fabrique de la «ville créative » s'accommodent fort bien du discours innovant et bohémien qui enveloppe une majorité de projets identifiés à l'urbanisme tactique (Mould, 2014; Deslandes, 2013).

Les fondements de l'urbanisme tactique, d'abord portés par les mouvements communautaires et activistes, ont été cooptés et instrumentalisés par des opérations privées ou publiques d'aménagement, ce qui représente aussi un changement intéressant dans les pratiques normatives plus traditionnelles. Ces solutions minimalistes et temporaires dans la fabrique de la ville représentent souvent un banc d'essai et un terrain d'expérimentations pour des interventions plus pérennes soutenues par les agences publiques de planification territoriale (Silva, 2016).
Ainsi, l'espace urbain est de plus en plus parsemé de microlaboratoires laissant parfois place à la participation citoyenne et instaurant un changement graduel dans les techniques et les moyens de mise en œuvre de certains projets d'embellissement et de revitalisation ${ }^{3}$. Toutefois, "être en phase avec l'échelle humaine » peut représenter un « raccourci intellectuel répandu qui est de croire qu'une échelle d'intervention réduite est garante d'appropriation, voire de participation citoyenne » (Banville, 2016,). Ce n'est pas le cas. L'urbanisme tactique, dans ce réflexe qui embrasse allègrement le do it yourself, n'est pas garant d'équité ni de justice spatiale puisqu'il est trop souvent récupéré et adapté à une multitude de motivations qui ne vont pas nécessairement dans le sens de l'intérêt collectif (Deslandes, 2013). Les classes créatives s'en réjouissent et les élus locaux applaudissent, mais très peu de recul critique est exercé sur la véritable portée sociale et la durabilité de ces projets.

Comme le souligne Silva (2016), un des défis majeurs de l'urbanisme tactique réside dans son rapport au cadre plus formel et normatif en urbanisme et en aménagement du territoire, et dans sa capacité à sintégrer aux mécanismes de planification existants. Il existe déjà des signes de cette intégration dans les stratégies de "prévitalisation" des friches et sites délaissés (mise en valeur temporaire et éphémère des espaces ou bâtiments vacants) ou encore dans les interventions de type intersection repair (mise en valeur de croisements et d'intersections de rues pour en faire des places publiques accueillantes).

This is not to deny that the idea of early civic improvers and later DIY urbanists grew out of different economic realities, involving different perspectives about aesthetics and order. It is only to draw attention to the cyclical nature of American grassroots activism, which can again be relied upon to initiate improvement when official channels fall shorts. (Talen, 2015, p. 146)

La multiplication des projets d'urbanisme tactique représente un défi pour la planification territoriale locale, et ce, en matière de cohésion globale, de cohérence avec le tissu urbain d'insertion de ces initiatives, de légitimité des actions et de leur pérennité dans le vivre ensemble à toutes les échelles. Si les relations deviennent effectivement de plus en 
plus poreuses entre les mouvements communautaires, les activistes et les gouvernements locaux, voire avec le secteur privé, il en ressort une reconnaissance et une pertinence sociale intéressantes dans l'analyse de la fabrique de la ville (Courage, 2013). La perméabilité entre les légitimités d'acteurs, les processus innovateurs de mise en œuvre des projets et l'acceptation somme toute généralisée des solutions envisagées fait de cet urbanisme tactique une impulsion de changement dans les mécanismes de planification plus traditionnels. Car, pour accommoder les projets d'urbanisme tactique, il faut contourner, assouplir et adapter les instruments normatifs que sont les règlements de zonage, de construction, de lotissement, etc. Il faut aussi assumer diverses formes de médiation entre les propriétaires fonciers, les commerçants, les résidents, les services de sécurité publique et les usagers des espaces concernés.

Banville (2016) le souligne avec justesse :

L'urbanisme tactique se déploie généralement dans les interstices de la ville, dans des endroits dits «sous-exploités». Nous ressentons un inconfort collectif par rapport à ces espaces illisibles - friches, terre-pleins, terrains vagues sous les ponts et échangeurs. Pourtant, ces espaces sont inhérents à la création de la ville capitaliste : les lourdes infrastructures de transport viennent avec leur lot d'espaces inhabitables, la production industrielle avec sa contamination. Plutôt que de remettre en question les valeurs qui sont à l'origine de ces espaces, nous $[\ldots]$ les soumettons aujourd'hui à un impératif de rentabilisation. Car parfois contre son gré, l'urbanisme tactique se révèle une solution idéale pour entrer à peu de frais ces espaces dans une logique lisse et productive. (Banville, 2016, s. p.)

Parmi les valeurs et les notions portées par l'urbanisme tactique, on retrouve une idée forte de reprise ou de réappropriation de l'espace public, pour le public et pour des usages publics inclusifs. Toutefois, pour certains projets, les frontières deviennent parfois vite embrouillées sur ce qu'est véritablement un espace public. Au-delà du mode de propriété foncière de l'emprise au sol ou des aménagements qui y sont localisés se pose la question de déterminer ce qui qualifie de «public» un projet quelconque. Est-ce le processus décisionnel dans la conception du projet qui doit être public? La nature des usagers visés par cet espace? Les modalités de prise en charge, de gestion et d'entretien de cet espace?

Dans l'imaginaire collectif, la sphère publique concerne tout un chacun, et le bien collectif relève de cet univers où le plus grand nombre, tout en diversité et en inclusion, est desservi par un projet déterminé. Dans la réalité, les visions et les conceptions de la dimension "publique » d'un projet peuvent varier sensiblement en fonction du statut des usagers et de leur appréciation des formes aménagées dans ledit espace public (Cicalo, 2013).

Finalement, il y a aussi une nécessité de distinguer les réflexions sur la forme par rapport aux fonctions de ces interventions. Le caractère expérimental de l'urbanisme tactique permet des ajustements et de la rétroaction, que les décisions soient collectives ou non. Les services offerts à la population et aux usagers de ces espaces sont tout aussi importants que la nature des aménagements. Un bon organisme de gestion (souvent présenté comme les «amis» d'un parc, d'un parcours, etc.) pourra dynamiser et consolider les projets ${ }^{4}$.

\section{Le cas montréalais}

Depuis plusieurs années déjà, la région montréalaise renforce sa réputation de ville festive et culturelle ainsi que de métropole cosmopolite et dynamique à plusieurs égards. Elle a su se positionner avantageusement tant auprès des clientèles touristiques que de sa population locale. Forte de ses indices élevés de qualité de vie et de ses nombreux quartiers à l'échelle humaine, elle a pris le virage de l'urbanisme tactique, et accueille toute une diversité de projets saisonniers et éphémères dans plusieurs zones de son territoire.

Une des organisations les plus connues en matière d'urbanisme tactique à Montréal, La Pépinière Espaces collectifs, est un OBNL créé en 2014 qui s'est illustré ces dernières années avec plusieurs projets, dont le Village au Pied-du-Courant, un site festif et multifonctionnel sur un terrain inutilisé en bordure de la voie ferrée le long du site du port de Montréal, les Jardineries sur le site du stade olympique, les jardins Gamelin, les quais Masson, la place du Marché, la rue de Dijon et le Café suspendu sur le mont Royal. Cette organisation présente sa mission ainsi : 
La Pépinière impulse et accompagne l'émergence et la gestion d'espaces de vie en communauté, afin de contribuer à l'amélioration de nos milieux de vie. Par la création et la gestion d'espaces publics pensés comme des lieux de rencontre et de partage, La Pépinière contribue à rendre les villes plus humaines, vertes et participatives. Afin de placer les communautés au cœur de notre démarche et de générer un élan collectif, nos projets sont coréalisés avec les acteurs locaux (citoyens, organismes, entrepreneurs, porteurs d'initiatives). Nées de la conviction que l'implication collective a le pouvoir de renforcer les communautés locales, nos approches visent à briser l'isolement social et à bâtir une société plus inclusive, équitable et résiliente. (La Pépinière - Espaces collectifs, 2019a)

Témoin des valeurs dominantes pour une ville à l'échelle humaine, l'organisation privilégie la dimension participative dans l'ensemble de ses projets. Elle est aussi chef de file à Montréal dans le mouvement qualifié de placemaking (aménagement d'espaces publics) $)^{5}$, et compte plus de 30 projets depuis sa création en encourageant l'engagement collectif et l'animation de ses espaces, de possibles qui « remodèlent l'expérience urbaine sur le mode du vivre ensemble» $(\mathrm{La}$ Pépinière - Espaces collectifs, 2019b, p. 8). L'organisation d'environ 30 employés anime trois types d'intervention: des laboratoires d'innovation, des projets de quartier et du soutien aux acteurs locaux et aux institutions. En 2018, son bilan, basé sur un chiffre d'affaires de 3 millions de dollars, se caractérise comme suit :

- Mise en place de 12 espaces collectifs au Québec;

- 25 consultations citoyennes;

- Plus de 200 partenaires et collaborateurs;

- 94 organismes ayant collaboré aux projets;

- Création de 120 emplois saisonniers et de 317 emplois bénévoles;

- Accompagnement de 156 initiatives portées par des citoyens et organismes locaux;

- Sept terrasses sociales et 1137 plantes mises en terre.

Au cours des trois dernières années, La Pépinière ne génère pas de nouveaux projets, mais améliore et bonifie ceux existants, et tente de valoriser davantage le volet animation des espaces déjà conçus. En effet, une fois les projets aménagés, le principal défi demeure de les animer avec une programmation et une appropriation réelle par les acteurs locaux, tout en assurant du soutien pour pérenniser les projets avec une structure de gestion opérationnelle à long terme.

Le contexte montréalais évolue rapidement en matière d'urbanisme tactique, avec d'autres organisations qui proposent des interventions qui se distinguent parfois de celles de La Pépinière par les modalités de financement et de mise en ouvre des projets. Par exemple, l'organisme Îlot 84, un OBNL fondé en 2015, est l'instigateur de plusieurs projets éphémères, dont Aire Commune dans le quartier Mile End, Nouvelle Vague dans le quartier du Vieux-Port, le Petit Montréal au square Phillips au centre-ville (une mesure de mitigation et d'animation du chantier de réfection de la rue Sainte-Catherine) et Green Haüs dans le secteur industriel Chabanel. La mise à contribution de commandites privées pour certaines initiatives d'Îlot 84 distingue d'ailleurs l'organisation de La Pépinière, qui préfère encadrer cette source de financement par des pratiques partenariales libérées des stratégies de placement de produits ou de mises en valeur excessives des bannières relevant de ces commandites. Le risque de voir glisser certains projets dans l'offre événementielle existante - une offre diversifiée, saturée et largement financée par des commandites privées - peut être perçu comme une atteinte aux valeurs fondatrices de l'urbanisme tactique citoyen et participatif.

Dans les faits, ce qui mérite d'être étudié et suivi de près au cours des prochaines années dans le contexte montréalais relève surtout de la diversité des organisations qui viendront instaurer de plus en plus de projets, et de la distinction des unes des autres dans les modes de financement et de gouvernance des projets. Surtout, il sera nécessaire de réaliser des études empiriques d'impact et d'évaluation de cette multitude d'interventions.

Plusieurs questionnements demeurent quant à l'évolution et à la véritable portée de l'urbanisme tactique, tant à Montréal que dans d'autres contextes géographiques. En quoi ce phénomène persistera-t-il et gardera-t-il son attrait pour plusieurs acteurs de la ville? Pourrait-il y avoir un effet d'essoufflement de ce qui pourrait être perçu comme une mode passagère par les critiques les plus cyniques? Quels sont les impacts significatifs 
de ces interventions dans la fabrique de la ville? Que restera-t-il comme héritage de ce mouvement?

Il y a encore matière à beaucoup de recul et de retours sur expérience dans le contexte montréalais. Les défis de la saisonnalité et d'un climat parfois capricieux; la récupération des valeurs inhérentes à l'urbanisme tactique par les élus locaux, les promoteurs immobiliers et d'autres agents de développement; l'éventuelle compétition entre des organisations fragiles économiquement et qui seront vraisemblablement plus nombreuses et en concurrence entre elles dans ce type de projet : voilà autant d'enjeux intéressants à documenter pour les années à venir.

\section{Conclusion}

L'évolution de l'urbanisme tactique est en adéquation avec les transformations récentes dans la fabrique des villes contemporaines et de celles à venir. Par un effet de balancier, des pratiques informelles plus anciennes dans les pays industrialisés et persistantes dans les pays en développement sont revisitées, remises au goût du jour et enveloppées des valeurs qui ont bonne presse : la durabilité, le recyclage et la récupération, l'éphémère et le transitoire, l'émancipation et la réappropriation dans le do it yourself, la mise en commun des ressources dans l'improvisation et l'innovation (open source), la participation citoyenne et l'événementiel.

Éthier (2017) propose d'analyser le phénomène dans sa composition spatiale, plus particulièrement dans la notion de connectivité qui renvoie à l'idée de mettre en valeur les marges et les interstices de la ville, à l’idée d'aménager avec une sensibilité accrue à notre univers communicationnel et à la nécessité de refonder le lien social dans la ville. L'idée est intéressante, car elle suggère que les analyses, les bilans, les retours sur expérience et les leçons à tirer en matière d'urbanisme tactique doivent être menés différemment. Effectivement, dans la réflexion sur l'urbanisme tactique, la littérature insiste beaucoup sur la forme des projets, la temporalité, les matériaux et les espaces interstitiels, mais très peu sur les finalités et le rationnel qui soustendent les motivations des citoyens et des usagers qui consomment ces espaces.

Nos villes connaissent une immense crise de sociabilité et une perte de repères dans les modes d'interaction sociale, qui sont vraisemblablement contaminés par les nouvelles dépendances technologiques. La question qui mérite d'être approfondie dans un éventuel agenda de recherche pourrait être recentrée sur les symptômes et les éléments qu'on peut extraire de l'urbanisme tactique comme option de rechange et comme stratégie de rétablissement d'une certaine forme de lien social. L'urbanisme tactique émane peut-être d'un besoin de ramener dans nos villes quelque chose de perdu ou d'oublié : cette forme de lien social vrai, senti, palpable dans l'expérience sensorielle du vivre ensemble. Notre capacité de se réapproprier les rues, les espaces de même que nos liens de proximité et de voisinage en dépend peut-être.

\section{NOTES}

1 Notons que certains projets sont parfois plus pérennes, car structurés pour revenir sur un mode saisonnier, de façon récurrente. Il existe aussi des évolutions et des transformations de projets temporaires qui deviennent peu à peu permanents, sous l'impulsion et la volonté des acteurs locaux et des usagers de ces espaces.

2 Les projets de park(ing) days et Reclaim the Street (fermetures temporaires de rues) sont des initiatives portées par le collectif REBAR, composé d'artistes, d'architectes, de designers et d'urbanistes de San Francisco, ville pionnière dans plusieurs initiatives d'urbanisme tactique.

3 La littérature fait beaucoup référence aussi à l'idée de placemaking (fabrique d'espaces publics), qui encapsule de bons aspects de l'urbanisme tactique, les professionnalise et les intègre aux outils traditionnels de planification urbaine pour les faire évoluer dans le sens d'une meilleure réponse aux besoins des usagers.

4 Nous aimerions remercier Jérôme Glad, cofondateur de La Pépinière - Espaces collectifs, pour ses commentaires et réflexions sur notre texte. Sa contribution nous a permis de nuancer notre propos et de prolonger notre réflexion.

5 Le placemaking est une approche misant sur un processus de cocréation d'espaces publics par une diversité d'acteurs locaux visant à promouvoir diverses formes d'appropriation d'espaces rassembleurs et innovants. 


\section{RÉFÉRENCES}

Acuto, M., Dinardi, C. et Marx, C. (2019). Transcending (in)formal urbanism. Urban Studies, 56(3), 1-13. https://doi.org/10.1177/0042098018810602

Banville, M.-S. (2016, 20 septembre). Les contradictions de l'urbanisme tactique. Le Devoir. Repéré à www.ledevoir.com/societe/ transports-urbanisme/480347/des-idees-en-revues-les-contradictions-de-l-urbanisme-tactique

Bradley, K. (2015). Open-source urbanism: Creating, multiplying and managing urban commons. Footprint, 9(16), 91-108. https://doi.org/10.7480/footprint.9.1.901

Cicalo, E. (2013). Designing public spaces and constructing public spheres: A manifesto. Dans S. Serreli (dir.), City project and public space (p. 221-235). Berlin, Allemagne: Springer. https://doi.org/10.1007/978-94-007-6037-0

Courage, C. (2013). The global phenomenon of tactical urbanism as an indicator of new forms of citizenship. Engage, 32. Repéré à https://engage.org/articles/the-global-phenomenon-of-tactical-urbanism-as-an-indicator-of-new-forms-of-citizenship

Deslandes, A. (2013). Exemplary amateurism: Thoughts on DIY urbanism. Cultural Studies Review, 19(1), 216-227. https://doi.org/10.5130/csr.v19i1.2481

Éthier, G. (2017). L’urbanisme tactique comme pratique spatiale de la connectivité? Connectivités, 125, 4-9. Repéré à https://id.erudit.org/iderudit/84826ac

Ferraris, F. S. G. (2016, 29 août). L'urbanisme tactique. [Billet de blogue]. Repéré à www.vrm.ca/lurbanisme-tactique

Finn, D. (2014). DIY urbanism: Implications for cities. Journal of Urbanism: International Research on Placemaking and Urban Sustainability, 7(4), 381-398. https://doi.org/10.1080/17549175.2014.891149

La Pépinière - Espaces collectifs. (2019a). À propos. Repéré à www.pepiniere.co

La Pépinière - Espaces collectifs. (2019b). Rapport annuel 2018. Repéré à www.pepiniere.co/rapport-annuel-2018

Lydon, M. et Garcia, A. (2015). Tactical urbanism: Short-term action for long-term change. Washington, DC: Island Press.

Mould, O. (2014). Tactical urbanism: The new vernacular of the creative city. Geography Compass, 8(8), 529-539. https://doi.org/10.1111/gec3.12146

Nédélec, P. (2017). De nouveaux mots pour de nouvelles modalités de fabrique de la ville? Initiatives citadines d'aménagement des espaces publics. L'information géographique, 81(3), 94-107. https://doi.org/10.3917/lig.813.0094

Paquot, T. (dir.). (2015). Les situationnistes en ville. Paris, France : Infolio.

Silva, P. (2016). Tactical urbanism: Towards an evolutionary cities' approach?. Environment and Planning B: Planning and Design, 43(6), 1040-1051. https://doi.org/10.1177/0265813516657340

Spataro, D. (2015). Against a de-politicized DIY urbanism: Food not bombs and the struggle over public space. Journal of Urbanism: International Research on Placemaking and Urban Sustainability, 9(2), 185-201. https://doi.org/10.1080/17549175.2015.1056208

Talen, E. (2015). Do-it-yourself urbanism: A history. Journal of Planning History, 14(2), 135-148. https://doi.org/10.1177/1538513214549325 